\title{
Using Concrete Scales: A Practical Framework for Effective Visual Depiction of Complex Measures
}

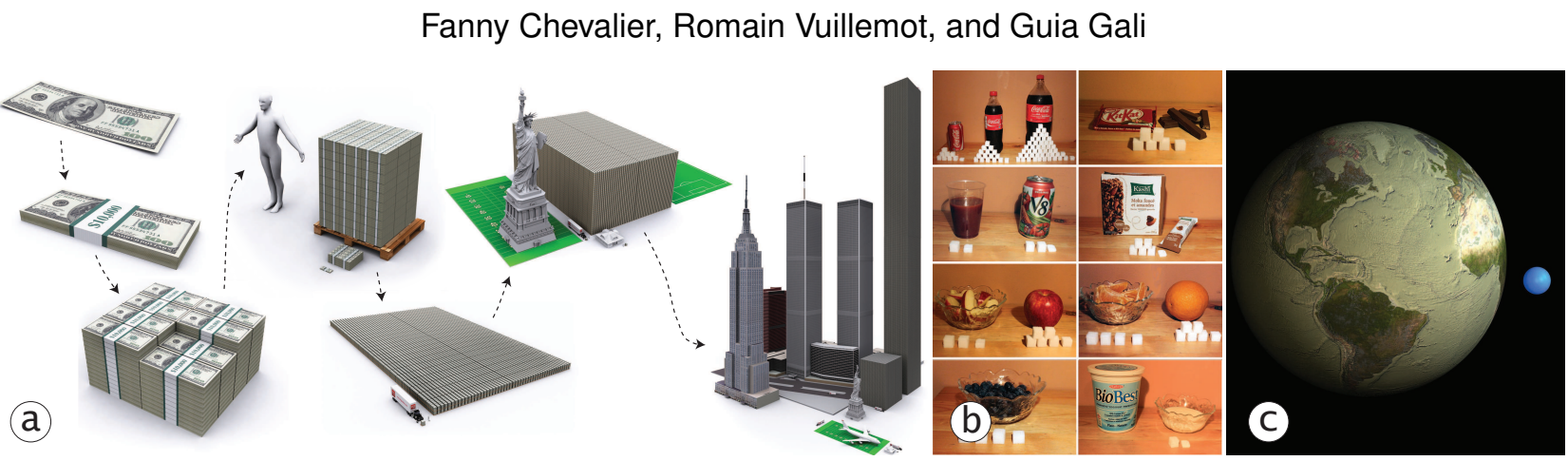

Fig. 1. Illustrates popular representations of complex measures: (a) US Debt (Oto Godfrey, Demonocracy.info, 2011) explains the gravity of a 115 trillion dollar debt by progressively stacking 100 dollar bills next to familiar objects like an average-sized human, sports fields, or iconic New York city buildings [15] (b) Sugar stacks (adapted from SugarStacks.com) compares caloric counts contained in various foods and drinks using sugar cubes [32] and (c) How much water is on Earth? (Jack Cook, Woods Hole Oceanographic Institution and Howard Perlman, USGS, 2010) shows the volume of oceans and rivers as spheres whose sizes can be compared to that of Earth [38].

\begin{abstract}
From financial statistics to nutritional values, we are frequently exposed to quantitative information expressed in measures of either extreme magnitudes or unfamiliar units, or both. A common practice used to comprehend such complex measures is to relate, re-express, and compare them through visual depictions using magnitudes and units that are easier to grasp. Through this practice, we create a new graphic composition that we refer to as a concrete scale. To the best of our knowledge, there are no design guidelines that exist for concrete scales despite their common use in communication, educational, and decision-making settings. We attempt to fill this void by introducing a novel framework that would serve as a practical guide for their analysis and design. Informed by a thorough analysis of graphic compositions involving complex measures and an extensive literature review of scale cognition mechanisms, our framework outlines the design space of various measure relations-specifically relations involving the re-expression of complex measures to more familiar concepts-and their visual representations as graphic compositions.
\end{abstract}

Index Terms-Concrete scale, scale cognition, visual comparison, graphic composition, visual notation

\section{INTRODUCTION}

As a result of the recent economic crisis, "trillion" as a monetary figure has become part of our daily vocabulary. Yet for such a large amount, how capable are we to mentally grasp its true value? To better comprehend this quantity, a popular graphic composition of 100 dollar bills stacked next to familiar objects progressively demonstrates the volume of money from 1 million dollars that is roughly the same size as a briefcase, 100 million dollars that occupies a pallet filled as tall as an average person, all the way up to 115 trillion dollars, which corresponds to a stack of pallets rising as high as iconic New York city skyscrapers [15] (see Figure 1a).

This process of visually relating complex measures with familiar objects from the real world results in what we refer to as a concrete scale. This type of representation re-expresses complex measures using magnitudes and units that are visually and kinesthetically experienceable, making them easier to grasp [24]. In the previous example, the volume of bills does not solely communicate exact monetary value

- Fanny Chevalier is with University of Toronto \& OCAD University. E-mail: fanny@dgp.toronto.edu.

- Romain Vuillemot is with INRIA. E-mail: Romain.Vuillemot@inria.fr.

- Guia Gali is an independant graphic designer and researcher. E-mail: guiagali@gmail.com.

Manuscript received 31 March 2013; accepted 1 August 2013; posted online 13 October 2013; mailed on 4 October 2013.

For information on obtaining reprints of this article, please send

e-mail to:tvcg@computer.org. but links the conceptually-hard-to-grasp value of 115 trillion dollars with experienceable notions of handling 100 dollar bills. This allows the observer to break down the scale through smaller comparisons of how much space various amounts of money occupy relative to familiar and tangible objects, which eventually helps in forming a more concrete mental model of scale for this value of money.

Concrete scales are important since they mainly rely on simple relations of complex measures to more familiar concepts, easing the cognitive load when trying to comprehend accurate, numerical values on an absolute scale. This makes concrete scales highly common and popular among educational and decision-making settings. From grasping the scale of the solar system to balancing a country's trillion dollar debt, it is extremely important for concrete scales to properly facilitate measure relations, from absolute to relative and back, in order to convey approximations as accurate as possible.

While there has been extensive research on how we understand or construct mental models of scale, most studies have strictly focused on representations along tangible dimensions such as space and time. Other types of relations, such as conversion and re-expression, have received very little attention from researchers, with infographics receiving even less attention despite its popularity and common use.

To address this lack of design guidelines for concrete scales, we introduce a framework that examines the design space of various types of measure relations, along with their visual representations. We achieve this through an extensive deconstruction and analysis of numerous graphic compositions collected online. We also provide a protocol as a guidance to designers on how to use our framework for the creation and evaluation of concrete scales, further illustrated with a scenario. 

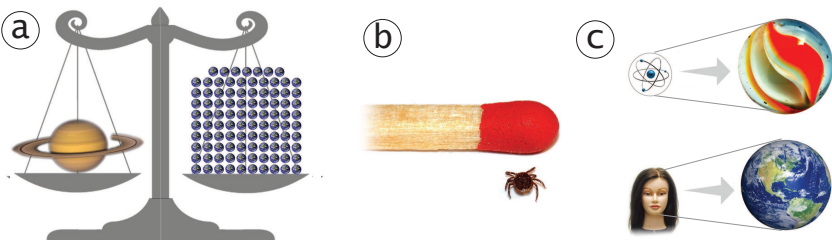

Fig. 2. Examples of concrete scales illustrating (a) unitization: the mass of Saturn amounting to 97 times the mass of Earth, (b) anchoring and adjustment: the match serving as an anchor to adjust the sense of scale of a tick and (c) analogies: the atom is smaller than a toy marble just as a human head is smaller than the Earth.

\section{BACKGROUND}

We begin by defining specific terminologies that we will be using throughout the paper, followed by a review of the challenges and cognitive mechanisms involved in interpreting complex measures. We then survey other related work that have tackled measure representations and visual comparisons.

\subsection{Terminology}

A measure is composed of a numerical value and a unit that quantitatively expresses a phenomenon according to a scale $[28,29]$. The numerical value of a measure conveys the magnitude of a phenomenonrelative to a chosen scale-and can range from small (e.g., 1 angstrom) to large (e.g., 100000 kilometers). A unit refers to a type of measurement ranging from the tangible (e.g., meter, dollar) to the more conceptual (e.g., calories, kilowatt). Units are employed to determine the scales which are used to quantitatively express a phenomenon. A scale is a system of ordered marks at fixed intervals that serves as a standard reference, linking the magnitude of a phenomenon to conventionally defined numerical representations of size [17].

\subsection{Challenges in Interpreting Complex Measures}

Interpreting measures involves the construction of mental models of scale in a process referred to in psychology as scale cognition or scale sense-a topic that has captured the interest of many researchers $[13,17,26,27,33,36,37]$. For the purposes of our framework, we limit our analysis to complex measures, which can be difficult to grasp for the following reasons: 1) the magnitude transpires beyond humanly experienceable proportions making it too mentally demanding to conceptualize with absolute accuracy [17], and/or 2) observers are unaccustomed to the scale due to a lack of knowledge with the defined unit of measurement. Scales associated with such magnitudes or units appear unfamiliar to layman observers due to their lack of regular visual and kinesthetic experiences relative to one's self, necessary in building mental models of scale. For example, despite the fact that we use money daily, it is almost impossible to conceive money in trillions of dollars or to determine what magnitude of calories are either too much or not enough when dealing with nutritional facts, without personal or professional expertise on the subject.

\subsection{Cognitive Mechanisms}

Scale cognition studies in psychology suggest that personal and professional experiences play a critical role in the conceptualization of scale $[17,26,36]$. For students of various schooling levels, scale cognition is more notably refined as they become exposed to a broader range of magnitudes and units through visual and kinesthetic experiences (e.g., traveling across a country or looking through a microscope) [36]. Jones et al. also found that experienced professionals, from physicists to engineers, comprehend scales through direct interaction with phenomenons of various magnitudes $[13,36]$.

Studies in psychology have also highlighted the importance of unitization, anchor and adjustment, and analogies in scale cognition. Unitization is an improvised-yet subjective-systematization of units where observers can break down scales, using a preferred new unit, into more specific and relatable chunks [13]. For example, the mass of the Earth can be used to re-express the mass of Saturn (or any other mass too big to grasp) by the numerical value alone.
Hence, we can state that it requires 97 Earths to amount to the mass of Jupiter (Figure 2a). In this example, the unitization process consists of redefining the unit (i.e., Earth) based on its inherent properties (i.e., mass). We then designate 1 Earth as a new unit for mass, making a more apprehensible scale for assessing measures [37]. Isotypes [23] are also loosely based on a type of unitization where pictograms are redefined as "tokens" to represent a given measure (e.g., one bike symbol as 100 bikers, or a bulb icon as 1 kiloWatt per hour), though the visual representations are more symbolic than basic unitization.

Anchor and adjustment uses tangible references as anchor points from which observers can make estimations through adjustment on a relative scale (e.g., "as far as...", "about the size of...", "slightly lighter than..."). An example is shown in Figure 2b, where the match serves as the anchor to asses the size of the tick beside it. A previous study noted that experienced professionals who deal with various scales daily often have recourse to anchor points to relatively adjust magnitudes and units, functioning as quick mental benchmarks in the construction of mental models of scale [13].

Analogies rely on pairwise analogies, by comparisons of the difference in magnitudes between one pair of objects with that of another pair of more familiar objects. In Figure 2c, atomic size is explained by comparing its size ratio to the diameter of a marble and creating a pairwise analogy with ratio of an average human head to that of Earth. Analogies have been shown to help in estimating linear distances [12], which is further substantiated in studies examining the different strategies people use to represent nanoscales [19].

As discussed in the introduction, visual representations can be helpful in conceptualizing complex measures when the above mechanisms alone are not enough to build accurate mental models of scale. The next section is a review of representations using different modalities.

\subsection{Representation of Complex Measures}

Absolute numerical values of extreme magnitudes and unfamiliar units may be difficult to comprehend instantly, which is problematic when the context of their understanding calls for decision-making [20]. The representation technique is therefore critical in communicating measures $[7,13]$.

Most relevant to our work is Nieman who refers to representations that use "the real world as a canvas for data visualization" as concrete visualizations [24]. Concrete scales build upon concrete visualizations where matter-though unseen has actual physical existence in the world-is made visible and depicted in context of a real-world scenario (e.g., $\mathrm{CO}_{2}$ emission visualized as a black cloud of pure gas diffusing in a city's streets). We extend Nieman's concept to measures that lack a physical counterparts, and examine scale sense in light of psychology research, from which the identified cognitive mechanisms can be leveraged as strategies for visual representations.

Animations such as Powers of Ten [5] or interactive graphics like The Scale of the Universe [10] and Scale Ladders [18] use zooming effects to progressively move from nanoscopic to cosmic distances. This continuous and progressive redefinition of the representation spacewhere the order of magnitude is made clearer by the addition of familiar objects (e.g, a hair, an ant) as anchors-aids in building mental models of scale along a continuum. As such, these tools have been proposed for educational and scientific purposes to facilitate conceptualization from relative to absolute scales with accuracy.

Walking scale models have been used to provide observers with a visceral feel for the vast scale of the universe. The Sagan Planet Walk [30] is a walkable 1-to-5 billion scale model of the solar system consisting of obelisks situated along a $1.18 \mathrm{~km}$ trail. Other works have mapped geologic time to walkable distances along customizable and familiar landmarks and routes on Google Earth to bridge abstract, imperceptible concepts of time through experienceable routes where the accuracy of human perception is higher [14, 26].

Temporal-aural-visual feedback representation has also been explored in nanoscopic scales through the accumulation of imperceptible elements across the diameter of a pinhead. The temporal aspect was found to be of significant aid to the observers but experiments have 
shown that without the visual modality, observers lost support for efficient anchor points in the construction of mental models of scales [31].

There is no shortage of graphic composition depicting complex measures but despite its abundance, little attention has been devoted to the analysis of various measure relations and visual strategies within it. To address this void, we have narrowed down our analysis to visual representations alone, which we then deconstruct and analyze for their efficiency as concrete scales (see Section 3).

\subsection{Comparison in Information Visualization}

Methods for effective comparison have been explored in visualization literature. Prior work include the study of comparison strategies specific to a particular data type such as tree structures [9], and flow datasets [39]. Gleicher et al. [8] proposed a more general taxonomy for comparison in information visualization, and identify three basic techniques for visually comparing two datasets: juxtaposition, superimposition, and explicit encoding (difference or time wrap). Javed et al. [11] introduced a design space for composite visualization views, where juxtaposition and superimposition are common strategies to Gleicher's classification. Other view compositions include overloading and nesting. Similar strategies are employed in concrete scales and we include an analysis of their usage in our context.

\section{Deconstructing Concrete Scales}

To better understand and analyze the various types of concrete scales, we collected and deconstructed up to 300 graphic compositions ${ }^{1}$. In this section, we discuss our procedure for data collection and the methodologies used in our analysis. The taxonomy of measure relations derived from this analysis is discussed in Section 5.

\subsection{Data Collection}

We collected our corpus by querying Google Images using multiple keyword combinations (e.g., infographics, scale, the size of, equivalent to, how much, as large as, what is the size of, how worthy is, etc.) and browsing through social networking circles, image-sharing websites, infographics blogs, and online newspapers such as the New York Times and the Guardian. The data collection process was conducted in parallel by all three authors and discontinued once a healthy convergence of unique graphic compositions were accumulated. Redundant representations across authors were discarded. Simple illustrations (i.e., images not mapped to data), and other images pertaining to concepts other than communicating measures of extreme magnitudes and unfamiliar units were not retained.

The corpus resulted to a set of 300 examples from domains as varied as Economics, Physics, Earth and Space Science, Biology, entertainment, nutrition, sustainability and so forth. A strikingly vast majority of these representations (293/300) was composed of pictorial depictions using real life objects. The semiotics of these objects in the context of concrete scales will be further examined in Section 4. Each graphic composition of our collection was deconstructed and analyzed using the framework below.

\subsection{Graphical Deconstruction}

Our deconstructive analysis is based on a recursive model for the decomposition of graphic representations defined by Englehardt [40]. In this model, a graphic composite consists of a set of graphic objects, which occupy and are contained within a graphic space, and a set of graphic relations, which are determined by the elements within the graphic composite itself. Graphic objects can be deconstructed recursively until its lowest level of deconstruction resulting to elementary graphic objects. Graphic objects can be interpreted through three levels of semiotic analysis: syntax, semantics, and pragmatics [16]. Syntax refers to the visual attributes of the graphic objects and the roles these objects play within the graphic composite. Semantics looks at the relations between graphic objects and the meanings these objects

1 The corpus of concrete scales that we have collected for, and used in our analysis is available at http://www.aviz.fr/concretescale
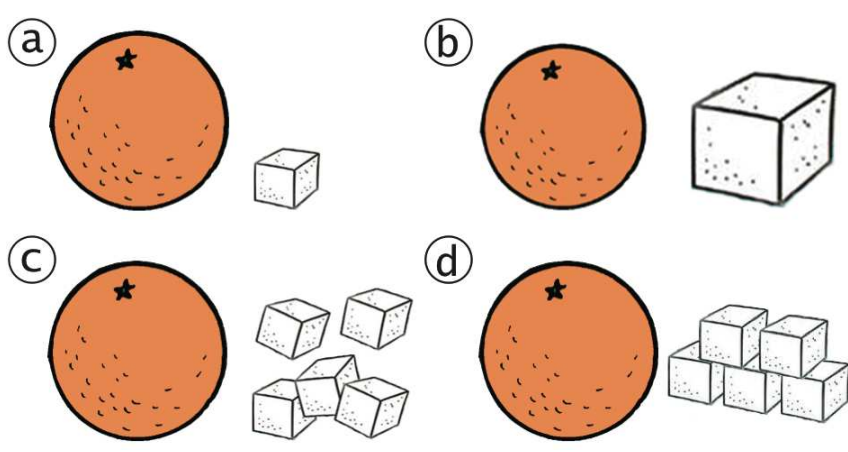

Fig. 3. Examples of graphic composites involving pictorial representations of real objects without notations. (a) The relative size of the orange and sugar cube is preserved: spatial attributes can be compared. (b) The relative size is not preserved: the orange-to-sugar cube relation is ambiguous. (c) The orange is juxtaposed to several sugar cubes, that may suggest that 1 orange is comparable to 5 sugar cubes. (d) Same as (c), rearranging the cubes to make them more easily countable.

infer within the graphic composite. Pragmatics refer to the connotations that are beyond the boundaries of semantic interpretation.

Semantics in particular identify the graphic object as representative of specific information, which is typically inferred through the interpretation of its relations with other graphic objects and the graphic space [40]. Object-to-space relations refer to spatial relationships between objects and their positions within a structured space. Object-toobject relations also include spatial relationships between objects (in the context of spatial clustering, linking, containment or nesting, juxtaposition, superimposition, ordering, etc.), as well as attribute-based relations between objects (e.g., relations involving variations in size, color, etc.). For the purposes of our framework, we have limited our analysis to pictorial representations of objects-in opposition to visual marks in charts - and their associated object-to-space and objectto-object spatial relations, which we particularly refer to as measure relations (further discussed in Section 5).

In the following section, we will take a closer look at semiotics in the context of concrete scales where pictorial representations of real life objects can be interpreted in various ways: be it from conveying different meanings to intentionally provoking certain emotions.

\section{Semiotics of ObJects in Concrete Scales}

Most concrete scales in our collection contain pictorial representations of objects borrowed form real life. Figure 3 displays an orange and sugar cubes juxtaposed in four different ways. Though all examples contain the same objects, they can be interpreted differently based on syntax, semantics, and pragmatics. For example, the sugar cube in Figure 3 could be seen as data-representative of a real sugar cube dimensions, functioning as a spatial ruler, from which observers can assess the real size of the orange and vice versa. Concrete scales that deal with distances or spatial occupancy — such as volume in Figure 1ause pictorial representations of the object's tangible counterpart as anchor points that frame the elements within the composite.

In figure $3 \mathrm{~b}$, the two objects' relative sizes are not preserved: the sugar cube is too big compared to the orange. Though the pictorial representation could still refer to a real orange and a real sugar cubeeven if only as a symbolic representative of another concept-their syntax is difficult to ascertain in order to associate it as a framing element. In this case, it is difficult to decipher object-to-object and object-to-space relations without explanatory notations or a more explicit visual composite that helps clarify the comparison logic $[8,11]$.

Figure 3c-d position the orange next to a collection of sugar cubes. Based on Gestalt's law of grouping, the sugar cubes represent a whole since they are spatially grouped together [41] with Figure $3 \mathrm{c}$ displayed more naturally than the organized stacks of Figure 3d. Due to the multiple instances of the sugar cubes, strict comparison of size and volume between the two objects is ambiguous. This reinforces a pairwise relation of orange-to-group of sugar cubes, which can be interpreted as an equivalence relation that possibly pertains to amount of sugar 


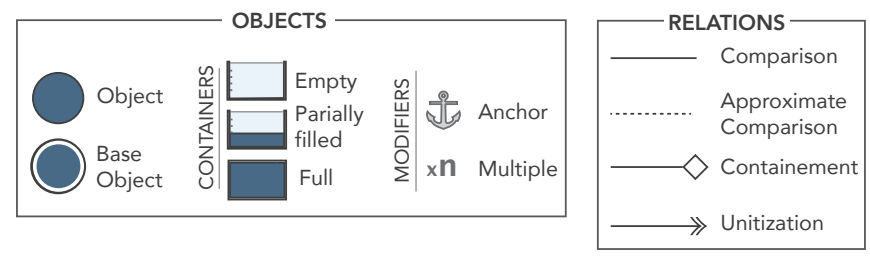

Fig. 4. Representation of object types and relations in CSML notation.

within an orange (i.e., "an orange is worth 5 sugar cubes"). Thus, the sugar cube becomes the new unit of measurement through unitization (see Section 2.3). Each cube plays the syntactic role of a "3-gram token" based on the idea that oranges contain an average of 15 grams of sugar. While equivalency in sugar amount is an obvious interpretation, it could also pertain to calories as a whole or other semantic meanings.

Besides syntax and semantics, pictorial representations can also connote other symbolic meanings based on cultural or personal experiences but can be intentionally chosen over other objects to capture and provoke desired impressions from the observer [35]. In our example regarding nutritional values, using a sugar cube as a token can be provocative when used to express, for example, the amount of sugar contained in sodas (Figure 1b). We could equally use an orange as a token to re-express the amount of sugar in these drinks, yet oranges are universally perceived to be healthier than sugar. This re-expressing of a can of soda as worth 10 sugar cubes versus 2 oranges invokes different sentiments and reactions. While the former aims to alarm consumers, the latter suggests a more positive message. This notion is further discussed in pragmatics in Section 7.3.

Non-object framing elements and notations are often omitted based on our data collection (only $56 \%$ of our examples included such marks or text), suggesting their insignificance in assessing measures in concrete scales. This observation is further substantiated by a study that found the inclusion of rulers and numbered lines does little to aid people's comprehension of nanoscales [18]. However, we have noted that in some cases the syntax and semantics of pictorial representations are not always clear, especially when an object representation can be interpreted in several ways: be it from corresponding to the real object itself, serving as a spatial ruler (anchor and adjustment), or serving as a token with a value (unitization). This is reflected in our corpus where cases with or without framing elements are evenly balanced.

Pictorial representations can also be interpreted with numerous physical properties borrowed from the real object such as spatial properties (e.g., width, height, diameter, volume), non-visible properties (e.g., weight, nutritional properties, energy power, storage capacity), the conventionally attributed (e.g., monetary value), or even symbolic properties based on cultural concepts or another. The variety of properties an object can possess widens their use in either anchor and adjustment or unitization contexts, making the assessment of measure relations complicated. We address this in the next section by establishing a taxonomy of object types and measure relations that deal with these issues, which we will then use as a framework for systematic decomposition of concrete scales.

\section{5 a taxonomy of Measure Relations}

Based on our literature review and a thorough analysis of infographics as described above, we derived a taxonomy of object types and measure relations involved in concrete scales. For the purposes of our conceptual framework, we also introduce a novel visual language that we call CSML (Concrete Scale Markup Language), similar to UML to encode these objects and relations (Figure 4).

\subsection{Object Types}

We have previously seen that objects contained in concrete scales can be of various natures and play various roles as they are involved in measure relations with other objects. When involved in a measure relation, an object refers to a single measure along one of its inherent properties for assessment (base) or as a reference (target). In our framework, we call a base object any entry object for which measure is to be assessed. Note that concrete scales can contain more than one base object at the same time.

We refer to particular types of objects that are fillable as containers. A container can embed other objects within its borrowed physical constraints, such as a plastic bottle filled with liquid or gas. A container's capacity (i.e., volume) can be graduated to enable partial filling (like a measuring cup) or possess translucence to reveal content (e.g., a glass). A container can be filled with different objects, thus inheriting different properties. For example, the same glass can present nutritional values based on whether it is filled with milk or orange juice. We also consider virtual space-filling areas and volumes as containers as they enable the grouping of objects into a single entity.

An object can be an anchor, when it serves as a benchmark to give a sense of magnitude. The anchor status is conveyed in CSML by adding a modifier icon to objects (see Figure 4).

Objects involved in concrete scales typically are representative of a tangible counterpart with proper spatial properties. In the context of concrete scales, these properties can be absolute with respect to the specific instance of the object, and therefore accurate (e.g., the Eiffel Tower is $320 \mathrm{~m}$ high) or more vaguely defined (e.g., the average height of a person varies depending on culture or personal experience).

\subsection{Measure Relations}

Analogical to Engelhardt's recursive model for graphic decomposition, we introduce measure relations, which are object-to-object relations involving comparisons, containment, and unitization of objects with regard to their measurable properties on a scale.

\subsubsection{Comparison}

Measure relations at the elementary level compare the magnitudes of shared property between objects. For instance, $\$ 10$ and $\$ 100$ bills share spatial and monetary properties. These pairwise comparisons result to a conclusion of equality, inferiority, or superiority. Our framework encompasses two types of comparisons: exact and approximate. Exact relations concern cases where magnitudes of objects can be accurately compared along a property (e.g., a $\$ 100$ bill is 10 times superior than a $\$ 10$ bill in terms of monetary value). However, such relations may not be conducive to exact comparisons when, for instance, the magnitudes are vaguely defined (e.g, the height of an average person). These phenomenons would fall under approximate relations.

Comparisons are by far the most prevalent relations that we found in our corpus ( $83 \%$ of the examples integrated at least one such relation), equally involving objects of which inherent dimensions can be extracted (e.g., iconic buildings, standardized objects and products such as coins, electronic devices, or deck of playing cards) and objects which dimensions are less certain (e.g., an elephant, a human, or a pencil). Comparison relations in CSML are represented with line connectors: plain when an exact comparison is possible, and a dashed line when the comparison is only approximate.

\subsubsection{Containment}

When objects are placed within a container, both objects become linked by a containment relation. Containers are common in concrete scales especially because they allow several objects into a single entity, which can facilitate comparison. In particular, we note that concrete visualizations, as defined by Nieman, primarily rely on the use of containers to portray otherwise impalpable volumes [24]. Additionally, there is a natural progression to using containers in the visual representation of small particle scales, like sugar grains or liquid, which are usually contained in the physical world in order to hold the matter in place. Other typical examples of containers include household items like spoons or cups - objects of which we are most familiar with.

About $8 \%$ of our data examples included containment relations, with more than half (15/26) involving loose matter filling physical objects like dishes or virtual geometrical shapes. Other containment relations include nested views, where a scaled version is nested inside a larger view, creating a composite visualization [11]. We discuss these further in Section 5.3.2. Containment relations in CSML are represented by connector lines ending with a diamond. 

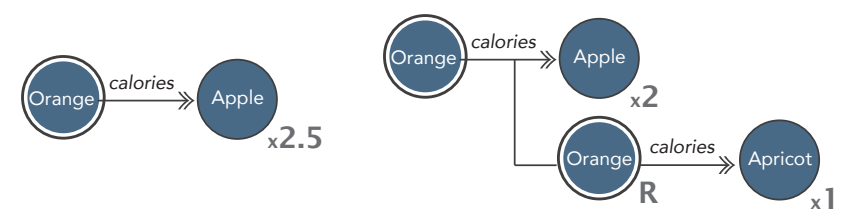

Fig. 5. Examples of unitization relations with remainder. Left: the remainder can be expressed in terms of sub-portion of the unit (resulting in a decimal ratio). Right: the remainder is recursively processed using a unit of a finer grain.

\subsubsection{Unitization}

Unitization, previously discussed in Section 2.3, consists of redefining an object as a new unit of measurement for assessing the magnitude of other objects in terms of this new unit. It results in relations of the form "A accounts for $n$ instances of B", where B is the new unit. The process of unitization therefore generates a set of $n$ objects B that, as a group, can be involved in turn in containment and comparison relations. Hence, not only does the process of unitization allow for the proportional assessment of $\mathrm{A}$ and $\mathrm{B}$, but it also creates a new instantiation of the object $\mathrm{A}$ in the form of a collection of another object that is potentially a better candidate for accurate assessment of magnitudes.

Unitization assists in computing the ratio between the object and the chosen unit. In the case the ratio cannot be expressed by an integer, a remainder (corresponding to a fraction) must be added to reach the balance. When possible, the remainder can be expressed in terms of sub-portions of the unit. The remainder can also be recursively unitized using a novel, finer-grained unit along the dimension. For example, an orange can either be re-expressed as two and a half apples, according to its nutritional values or two apples, and one apricot. Figure 5 shows these two relations in CSML visual notation.

About $16 \%$ of our corpus included unitization relations, all of which re-expresses non-visible units such as weight, calories, or time, using typical objects that are usually associated with these dimensions (e.g., an elephant is well known to be heavy).

Our framework considers unitization in a broader sense than previous work has done when examining object-to-object relations [19]. We formalize unitization as a recursive process for further breaking down remainders into other types of units. Though the unitization with remainder is a valid strategy conceptually, we only encountered a few examples in our database. We hypothesize that the reasons may lie in the complexity introduced by a larger variety of displayed objects used in the relation (e.g., how to form the accurate groups of objects for the comparison?), and an overall preference for round numbers.

\subsection{Strategies for Concrete Scales}

We build upon studies that have previously examined strategies that people undertake to represent nanoscopic objects [19]. We generalize and extend this list beyond space to encompass dimensions that are not directly visible (e.g., weight, nutritional value) along with conventionally attributed properties (e.g., monetary value).

Not surprisingly, the most common strategies employed by concrete scales ${ }^{2}$ that we have identified in our analysis are visual transcriptions of the cognitive mechanisms as discussed earlier in Section 2.3, which are anchor and adjustment (through direct comparison) (83\%), unitization (16\%), and analogy (4\%). Anchor and adjustment and unitization involve a single measure relation between objects, as depicted in Figure 6. In contrast, analogies involve a combination of such relations. In both cases, objects are displayed either in juxtaposition (the most popular, accounting for $82 \%$ of the relations) or superimposition, each of which being prevalent layout strategies for comparison in visualization [8]. Another strategy typical to representing cosmic and nanoscopic scales is the zooming inwards and outwards effect $(2 \%$ of our corpus) [19], all depicted using a nested view, where a scaled version of a partial view is embedded in the larger picture [11].

Here, we discuss analogy, zoom, and a few other strategies that combine measure relations using CSML visual notation (see Figure 6).

\footnotetext{
${ }^{2}$ Note that the same representation can make use of several strategies.
}

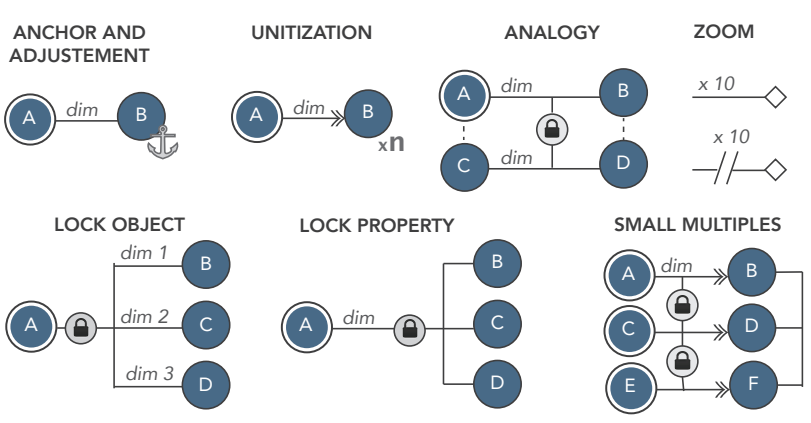

Fig. 6. Examples of popular strategies for concrete scales.

\subsubsection{AnAlogy: Simultaneous Pairwise Comparison}

As described by Ma [19], "Analogies convey size relationships by comparing the size difference between a pair of objects, the target, and another pair of more familiar objects: the base." Analogy is one of the cognitive mechanisms that contribute to our construction of scale representations (see Section 2.3). Hence, it is not surprising that we find them in concrete scales, though not that often (about $4 \%$ of our corpus use analogies). Using our framework, an analogy consists of two pairwise comparisons, which can be exact or approximate. In this situation, comparison relations are constrained in a sense that the dimension and the magnitude have to be the same. In other words, analogies build on equivalences between two pairs.

\subsubsection{Zoom: Progressive Adjustment and Containment}

When the magnitude is too large to both see details on a relative scale while having an overview of the absolute scale, a zoom effect is often used. When the tools are dynamic, the zoom effect is executed through animation [5]. On paper representations of nanoscopic and cosmic scales, the zoom effect is represented as side-by-side views where one view is a magnified portion of the other. This magnified portion can be of a zoomed in or zoomed out section, which is made even explicit by outlining and linking the two views graphically.

The zoom effect is therefore similar to a comparison relation where the magnification factor enables scale understanding. The zoom can also function as a container relation where the zoomed in part of the object is part of larger zoomed out object (i.e., nested views [11]). Hence, in addition to depicting magnitude relationships, a zoom effect also exhibits pairwise containment relationships between the graphic composite's objects.

\subsubsection{LOCK: One Element or Measure to Compare them All}

Lock strategies provide another interesting approach to concrete scales. The general idea builds on locking the possible relation of an object to a specific type as a constraint. Constraining of only the relation ("equals to") allows for the exploration of equivalent conversions along dimensions. Locking on a dimension and magnitude (e.g., a comparison relation on the form of "is equal to, on a caloric scale") allows for exploration by facets, yielding to a collection of objects which all share the same properties. We found the lock strategy is as popular as the zoom one in our corpus, with $4 \%$ of the collection (14 images).

\subsubsection{Small Multiples: Build a Collection}

Juxtaposing multiples of small elementary objects aid in establishing a concrete scale through the use of a singular measure relation. Unitization is the most common practice of this strategy (11 out of the 28 cases), of which the sugar cubes in Figure $1 b$ is an example.

Many other strategies can be built by combining various measure relations. For the purpose of our framework, we have only reviewed what we have found to be most popular during our analysis. As far as our experience goes, all strategies can be deconstructed in terms of objects and measure relations as introduced in our framework and in the following section, we will review an example of such deconstructions on a series of graphic compositions that we have collected. Then, we provide guidelines on how the framework can be applied in a design process for the creation and analysis of concrete scales. 


\section{Deconstruction of Concrete Scales}

We thoroughly investigate seven graphic compositions-that span across the previously introduced taxonomy-based on the concrete scale's objects, relations and strategies. In the following, we outline each infographic's visual design and application domain, accompanied by its CSML diagram depicting the composition's conversion logic.

\subsection{Scale of the Universe}
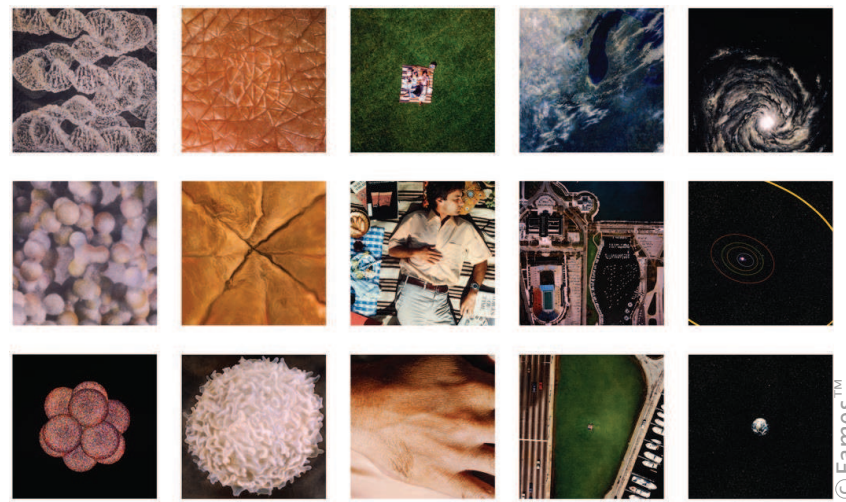

Fig. 7. Sequence from Powers of Ten (Eames $\left.{ }^{\mathrm{TM}}, 2010\right)$ zooming from human (center) to cosmic scale (right) and nanoscale (left) [5]

Powers of Ten [5] shows the relative scale of the Universe by connecting magnitudes of nanoscopic to cosmic distances, through a progressive zooming effect (Figure 7). It is considered the flagship example for concrete scale after its completion in 1968 as a TV program for educational purposes. The animation begins with a camera zooming out from a real-life scene-a picnic scenery at $10^{0}$ meter)-in a constant speed rate of one power of ten unit per 10 seconds. Once the observable universe is within the field of view at $10^{24}$ meters away from the picnic scene, it starts zooming back inwards towards the picnic scenery concluding at a nanoscopic scales where quarks in a carbon atom's proton are visible at $10^{-16}$ meters. The smooth transitions between levels give the observers an overall sense of the scale as a continuum: there is no leap from one measure to another-only graphical marks that note the field of view of the camera at every power of ten. The result of the decomposition using CSML is depicted in Figure 8.

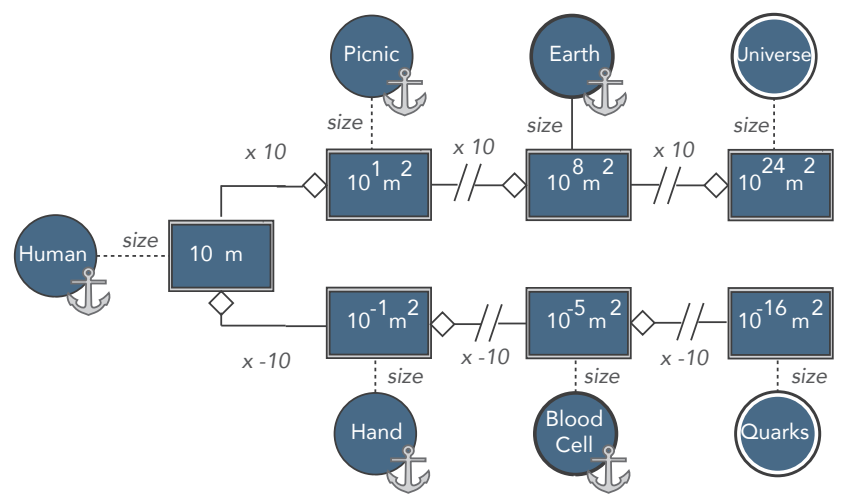

Fig. 8. Schema CSML decomposing Powers of Ten animation (Figure 7).

Powers of Ten's journey begins at a familiar level by establishing the initial scale in a picnic scenery among people, a magnitude of scale that we experience everyday. It then connects the anchor to space (object-to-space) as the origin of a vertical axis ruler that will be explored in both directions through progressive zooming. During this axis exploration, graphical marks are added at every power of ten units for object-to-object connections to the anchor. The zooming animation reinforces the object-to-object connections, with a physical time duration of 10 seconds between each objects. Powers of Ten is a typical example of the Zoom strategy introduced in section 5.3.2.

Powers of Ten is a dynamic adaptation of Boeke's essay Cosmic View published in 1957 [4]. Several other films have also used the same idea and techniques, including Cosmic Zoom produced by the National Film Board of Canada (1968) [34] and Cosmic Voyage
(1996), which was nominated for the Best Documentary Short Subject Academy Award [21]. An interactive application (called Cosmic Eye) has also recently been released [25]. All of these demonstrate the great amount of attention Boeke's concrete scale has received from researchers and the public.

\subsection{How Much Water and Air?}

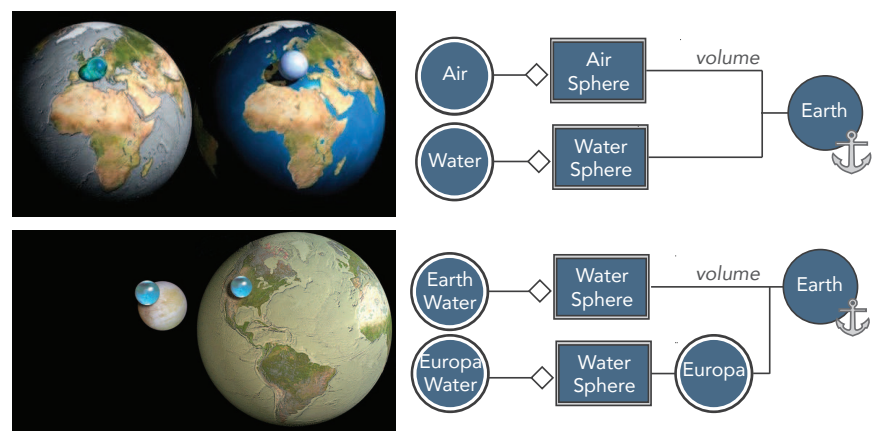

Fig. 9. All the water and all the air (top, A. Nieman, 2010) compares the volume of air and water with that of Earth [24]. All the water on Europa (bottom, K. Hand, J. Cook, H. Perlman, 2012) features the volume of water on Earth and Jupiter's moon Europa [22]

Figure 9 depicts illustrations of the volume of water and air on Earth and Jupiter's moon Europa-represented as spheres resting on the ground of the globe and the moon Europa. All the water on Earth, including oceans, fresh water and clouds $\left(1.4\right.$ billion $\left.\mathrm{km}^{3}\right)$ would fit in a sphere measuring $1,390 \mathrm{~km}$ across. The sphere containing all the air on the atmosphere measures $1,999 \mathrm{~km}$ across. The volume of water on Europa is estimated 2 times as much as the volume of the Earth oceans [22], corresponding to a $1,750 \mathrm{~km}$ wide sphere-lying on Europa's ground, in juxtaposition to the Earth and its water sphere. While the graphics uses both Earth and Europa as a background, the only Earth serves as an anchor for object-to-object volume comparison, since it is unlikely that Europa is familiar enough to laymen.

As we can see on the CSML diagrams of the two concrete scales, both representations build on containment relations into spheres to make it possible to manipulate volumes (water and air) in comparison to the original form of the Earth and the moon Europa.

Nieman suggests that "it is not just the familiarity with the globe that provides a sense of scale; it is also the observer's experience of travel" [24]. Since different people have different experiences, he proposed three versions of All the water for different audiences, where the sphere is respectively centered on Europe, North America and Asia.

\subsection{Sugar Scales}

Nutrition facts often require extra effort to decipher amounts and units that are not always easy to relate to with one's needs. As such, they are good candidates for concrete scales. Figure 10 depicts three alternative designs using sugar (cubes and grains) as the reference scale.

Figure 10a is a close-up version of the tables of Figure 1b, where calories are re-expressed using sugar cubes as the unit in a UNITIZATION relation. Sugar cubes are organized into stacks acting as virtual containers. Each food and drink declines by different quantities according to its container or its typical portion size, which are then converted into 15-calorie sugar cube stacks of, e.g., 9 cubes and 3/4 for a $35 \mathrm{cl}$ can of soda (139 calories). Figure 10a shows the conversion steps for sodas with the mechanism remaining identical to other cells of Figure 1b. The comparison holds between an object-to-object relationship between the different sugar stack containers. Since conversions from calories to sugar cubes does not always result in a rounded number, broken pieces of sugar cube show the remainders.

Figure $10 \mathrm{~b}$ presents an alternative by filling a container with a continuous volume of sugar grains instead of discrete sugar cubes.This conveys the nutritional value of drinks through the filling of plastic bags (containers) with sugar grains for the object-to-object relations within the bags. Since the bags all have the same capacity, the volume of sugar can be visually compared across and relative to the drink packages. The CSML diagram for sugar grains is roughly similar to the 

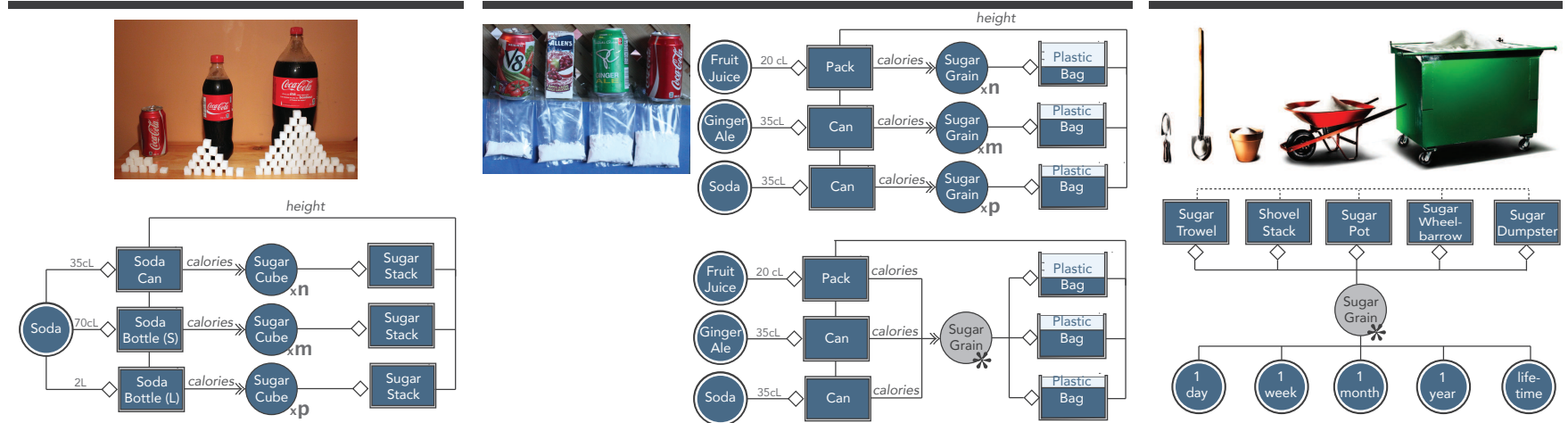

Fig. 10. Examples of concrete scales that use sugar as a representative scale for measurement. (a) Sugar stacks (see Figure 1b) use sugar cubes as a unit, while (b) Rethink your drink (adapted from anonymous [6]) employs plastic bags as containers for sugar grains to re-express the amount of calories in foods. (c) Is sugar toxic? (Kenji Aoki, 2011) is another example of containment relations, where garden utensils are filled with sugar grains to facilitate the estimation of one's average consumption of sugar in different time spans ranging from 1 day to a lifetime.

one with sugar cubes. To formalize repetitive use of the same relations, CSML also proposes a factorized notation (bottom diagram).

Figure 10c shows the average consumption of sugar across various time spans by filling containers with the corresponding volume of sugar grains. Since the volume grows exponentially, extra containers are added using objects from the same family (e.g., gardening utensils) that are large enough to be filled with the ever-increasing volume of sugar. The CSML diagram differs from the previous example since the containers cannot be accurately compared to each other due to the objects' varying volumes and shapes.

\subsection{US Debt}

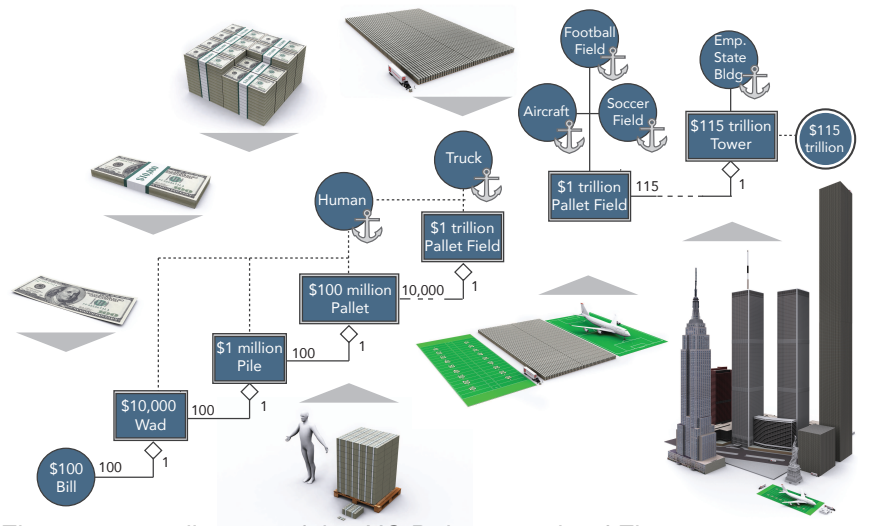

Fig. 11. CSML diagram of the US Debt example of Figure 1a.

US Debt [15] is a series of CGI data graphics that displays various amounts of money, ranging from 100 dollars to the 115 trillion dollar US debt using successive piles of $\$ 100$ bills (see Figure 1a).

Figure 11 depicts the CSML conversion diagram following an ANCHOR and ADJUSTMENT scheme, with the initial landmark being the $\$ 100$ bill quickly transforming into a $\$ 10,000$ wad. In-between measures are then added for adjustment (alternatively with a factor of 10 or 100), either to reach a specific intermediate or final amount (e.g., rounded values like 1 million dollar), or to be converted into a specific object (e.g., a human-sized pallet of bills to serve as a comprehensive landmark). This is visible in the diagram with the 1 trillion dollar threshold (corresponding to a field of pallets) further emphasized as it appears twice with no adjustment in-between. This inconsistency is aimed at giving observers a break and to visually adjust the spatial area in order to fully grasp the spatial occupancy of such a value as the view changes and new anchors are introduced (e.g., human, aircraft).

The same author has produced other similar graphics for European countries' debts, this time using local landmarks (i.e., $100 €$ bills) and anchors (i.e., the Eiffel Tower). Other representations of debts have been introduced, such as US debt as a journey [1], where each mile corresponds to a $\$ 5.8$ million increase in debt, allowing to estimate how far various US administrations have 'traveled' across the country.

\subsection{Discussion and limitations}

We explored seven case studies using our taxonomy and the CSML notation. From the systematic dissection of our corpus emerged a perception of concrete scales as an iterative relation-to-relation approach, forcing a closer inspection of individual objects and relations, and hence the underlying mechanisms. We leverage this methodology as a basis for the creation and evaluation of concrete scales, which can serve as a tool for more informed design practices in this domain. The next section outlines practical guidelines for this methodology.

Despite the variety of application domains, we found that our framework is suited for the deconstruction of a wide range of visual compositions. However, we also found some limitations to our framework, especially when the objects involved in Unitization are used to convey non-visible measures that are difficult to decipher at first inspection (e.g., planet weight in Figure 2a). In such cases, additional indications are often necessary (e.g., the physical scale in the background) to make it explicit what property to use for comparison. Using SMALL MULTIPLES is one approach to overcome this potential ambiguity (e.g., Figure 1b), but this strategy does not fully address the problem. These difficult cases aim at expressing a non-visible measure using a physical object-whose relative size may or may not reflect reality - which potentially leads to misinterpretation. This category of concrete scales falls into the category of isotypes [23], which are special cases that continue to challenge our current framework.

\section{Construction and Analysis}

Based on our extensive review of graphical compositions and previous experience as designers, we propose a series of guidelines that we hope can help in assessing the robustness of concrete scales. These guidelines are organized in a question and answer format that designers can use as a tool for both the evaluation and creation of graphic composites through an iterative relation-to-relation approach. Additionally, we outline possible challenges and difficulties, of which we do not always have a definite and optimal solution for. Regardless, we still provide discussion and viable directions for a workaround.

\subsection{Proceeding by blocks}

When given a measure to assess, two strategies can be employed: a bottom-up approach where we can progressively build CSML relations (or blocks) on top of a measure until the object represents an experienceable measure; or a top-down approach beginning with a familiar object involved in relations, and deducing from this until we can link the object with the measure to assess. We propose a series of questions that will attempt to assist designers in making informed choices at the local level of a block while ensuring the coherence of the concrete scale as a whole. The following introduces a general bottom-up process where the analysis and construction begins with the complex measure to assess. The top-down approach follows a similar but inverted process. We will then illustrate the process with an example. 
Q1. What makes the measure difficult to assess? We argue that the primary goal of an effective concrete scale ultimately consists of the re-expression of complex measures using physical objects, whose visual properties can be used to estimate the quantity of how much space these would occupy in the physical world. A measure is complex because of either:

Unfamiliar unit. In essence, the first step is to re-express an unfamiliar unit in terms of spatial magnitude assessment through a unitization CSML block. See (Q2).

Extreme magnitude. Anchor and adjustment, analogies and unitization are all suitable mechanisms that allow for a comparative assessment. We further follow up on this question in (Q4).

Q2. Is it a good unit? The difficulty of unitization lies in the search for an appropriate object to represent the unfamiliar unit (e.g., a teaspoon of oil is a better representative for calories than an egg, which is better known for its protein content). Objects with universally attributed values (e.g., a coin and its monetary value) are ideal candidates when they exist. Finding appropriate objects that will be able to stand on their own without further explanation is equally challenging for complex units, such as derived units like frequency and decibels. Certain situations are even more troublesome when the correct interpretation of the object depends on multiple factors like culture and the viewer's personal experiences. While in some cases the use of small multiples partially addresses this problem, the criteria for what makes a good unit in the unitization process remains open. Hence, we recommend designers to conduct surveys with some viewers to assess the robustness of their choices. Moving on to $(\mathbf{Q 3})$.

Q3. Can/shall the physical property be used? Once the object for unitization is determined, the next step is to convey the quantity that amounts to the initial measure. To this end, a possible strategy is to switch to the physical space and provide tools to help estimate the spatial occupancy of the collection of tokens (e.g., Figure 1a), either by using containers (see (Q6)), or by solving the problem of extreme magnitude (see (Q4)). In certain cases, using the object's physical property may not be appropriate, especially when none of the inherent properties of the physical object is borrowed (i.e., isotypes). In such cases, our experience suggests the use of framing marks or text to disambiguate the measure involved in the comparison.

Q4. Which technique to use? Previous works have provided insights on the preferred techniques in the representation of spatial relations [19] with anchor and adjustment being the most popular, followed by analogies - a trend also reflected in our collection.

Anchor and adjustment. The first step is to identify a more familiar object that will serve as the anchor in a comparison relation. See (Q5).

Analogies. Like anchor and adjustment, analogies also rely on comparison relations though in this case, three additional objects are needed to form two pairs involved in comparison relations. The first pair should relate the object of extreme magnitude to a familiar object. The second par, serving as the reference for the analogy, must involve familiar objects whose relative magnitude is comprehended well by the viewer. The difficulty here lies in the search for objects with relational constraints in order to depict a meaningful analogy. While analogies provide a good sense of proportional reasoning, it falls short in conveying absolute scales by failing to contextualize the two analogous pairs along an absolute continuum. This further clarifies why anchor and adjustment is the overall preferred approach.

Unitization. Unitization can also be used to convey a measure of extreme magnitude by breaking it down into more relatable chunks by using a preferred unit. As the accumulation of objects used as a unit increases, these objects must be kept organized into a coherent volume to remain easily countable, or enclosed into containers (see (Q6)).

Q5. What anchor(s) to use? We identified several concerns that play a role in the choice of anchors. We break them down as follows:

Q5-a. In what spatial dimension(s)? Spatial measures can be one, two or three-dimensional. The anchor should first be considered for its familiar and unambiguous properties along the relevant dimensions of the measure to be assessed (i.e., height, surface, volume). Several anchors can also be used to complement one another, for instance, the area of a soccer field and the height of an iconic tower can be combined to eventually amount to a volume (e.g., Figure 1a). As a rule of thumb, the combination of anchors, whose measurements are most familiar, should take prominence over a single object that is less familiar or with an irregular shape. Basic geometrical shapes also make good candidates for manipulating volumes (e.g., the spheres in Figure 9).

Q5- $b$. In what magnitude? As the distance between two magnitudes increases, the accuracy of our estimations diminish [27]. This suggests that a good anchor should be familiar enough to have close spatial properties to that of another object in order to retain precision. This would also ensure that both objects will fit within the same real estate to prevent visual unbalance.

Q5-c. Is the anchor of an extreme magnitude itself? Anchor and adjustment lessens our grasp of absolute scale by limiting our comprehension along a relative scale [36]. While relative comparison is important (Q5-b), it is equally significant to provide tools for contextualizing such measures on an absolute scale. On this note, closest anchors to human size will be the most comprehensive, yet may still be too removed from humanly experienceable phenomena. Additional landmarks must be used to establish a continuum between human-size representations and the closest, yet still extreme, anchor. Hence, the process is to iterate on (Q5), using the latest anchor as the measure to be repeatedly assessed. (Q5-d) touches on coherence of a complete set of anchors while (Q5-e) addresses precision and readability.

Q5-d. Is the set of anchors familiar and coherent? When more than one anchor is involved in the representation, a good variety and consistency should be used. This is especially true when addressing broader audiences where discrepancies between anchors have been found to cause confusion [19]. The target audience and cultural differences should also be kept in mind when choosing anchors.

Q5-e. Is the concrete scale readable? Working with absolute scale eventually requires a change in the representation's zoom factor, particularly when distant landmarks are no longer legible. A proven technique to overcome this problem-while ensuring comprehension in both relative and absolute scales - is to use a zoom technique $[5,19]$ to adjust the representation space to the necessary magnitude. In concrete scales, this is typically achieved through nested views [11], where a new view of a different scale is displayed within the context of the previous one. Visual marks can indicate the scale relationship or designers can choose to present a progressive narrative using several images, each of which presents a different scale (e.g., Figure 1a).

Using larger sets of anchors at both the relative and absolute scale has other merits over using a single anchor or analogy. Progressive techniques help diminish fragmentation in scale cognition-an issue that becomes more apparent when classifying objects on an absolute scale with a lack of experience of in-between phenomena [17, 36]. As a guideline, we suggest adding as many anchors as necessary to ensure a continuum between familiar measures and those of extreme magnitudes. The more observers are exposed to potentially new anchors, the higher the odds of enriching their own repertoire. This increases the chance of recognizability (Q5-d) since many anchor points are not necessarily universally identifiable and accurately measurable.

Q6. When and what containers to use? Containers can be useful in adjusting units, especially when several instances of the same object are used to equal a measure (see e.g., the gradation from a single bill, to a wad, to a pallet of bills in Figure 1a). The guidelines for choosing a container are similar to those for choosing an anchor (Q5-c): if a container is too small (or too large) to convey a certain quantity, it can be grouped into a larger container (or broken into smaller ones) to adjust to the scale of the comparison relation. The same rules of continuity and consistency for anchors (Q5-d) also applies for containers.

This set of questions informs the design and analysis of concrete scales in terms of which technique and objects can be chosen. Section 7.4 illustrates how to use the aforementioned block-to-block process in a scenario. Two additional aspects in the design process are discussed in the following section. 


\subsection{Layout Considerations}

We have observed that traditional methods for comparison and composite views apply to concrete scales. In particular, juxtaposition seems to be preferred for pairwise comparisons of volumes, or comparison along a single dimension, while superimposition best suits comparison of surfaces [8]. Nested views [11], also relevant to concrete scales, should be used for containment and zoom relations.

We know from Gestalt that comparison is facilitated when objects are grouped and organized in coherent shapes. With unitization, there is a potential that many instances of the same object used as a unit may have to be depicted. Visually arranging these objects by aligning or stacking them in an organized manner allows for a better distinction of the different groups to be considered in a comparison relation (e.g., Figure 3d). More importantly, the methodized layout also makes it easier to count how many objects compose the groups. Note that the way objects occupy space will have a potentially significant impact on their numerosity [2] — a bias that may be leveraged to impress the observer but may substantially impair the accuracy of the scale.

\subsection{Pragmatics}

Bias in perception could be intentionally leveraged to make a stronger point on certain facts. Its apparent power in capturing people's attention and memorability can help communicate the gravity of otherwise impalpable phenomena and how it impacts their lives. The choice of objects in particular for anchor and adjustment or unitization can be subjectively chosen, especially when the intent is, for example, make the magnitude of a phenomenon appear even larger than it truly is to alarm the public. Even though we strongly recommend against the abuse of such practice - as it could impair an observer's sense of scale-designers can exploit such tools for purposes involving memorability, familiarity, impressionability and engagement [3, 35] e.g., to sensitize the public of certain issues.

\subsection{Scenario}

We illustrate the use of our framework in a simple scenario. Lisa, a graphic designer working for the Keeper newspaper, has been asked to provide a visual representation that contrasts the US debt with the average yearly salary of an American in reaction to a major increase in income taxes. She identifies that she should start with a unitization (Q1), and finds that the debt represents about 1 year of work in average salary for roughly 3,000 people. With the measure of the unit constrained, she needs a physical counterpart to represent this value (Q2). She faces a problem of isotypes, since a yearly salary does not translate into an existing object. For the lack of a more direct physical counterpart, she decides to use a human (worker) to represent the average yearly salary.

The next question pertains to physical space $(\mathbf{Q 3})$. To cope with the large number of people, she has a choice between packing people in an organized space (Q4), or putting them into containers, such as a bus or an aircraft (Q6). Using buses that fit 60 people, she calculates that she would require 50 vehicles to fit all the workers. As she considers the pragmatic aspects, she decides that using containers drastically reduces the number of objects for the final relation. She prefers to keep people as a unit, which she believes will be more impressive and comprehensible than using a container, of which the capacity can be misinterpreted depending on the reader's experience.

Lisa tries to address a problem of extreme magnitude (Q4), as she needs to convey spatial occupancy of 3,000 individuals. She finds out that the average height of a human is 5'10 $(1.70 \mathrm{~m})$. Stacking all of the workers amounts to $5,100 \mathrm{~km}$ high, which she knows to be approximately the altitude of Kilimanjaro. While thinking of juxtaposing a human stack next to the mountain, she identifies two major flaws. First, despite its stature as an iconic figure, it is unlikely that readers will be aware of the altitude of Kilimanjaro. Second, it is uncommon to think of a crowd of human beings in stacks, but rather in terms of land surface they occupy. She discards the stack idea and explores how large a surface the crowd would occupy if organized on a grid using $1 \mathrm{~m}^{2}$ tile per person: this results to a $50 \mathrm{~m} \times 60 \mathrm{~m}$ area. She then looks for a good anchor to use as a landmark to help assess this surface (Q5).
As she considers other breaking news to appear in the next issue of the newspaper, she finds out that a popular football player reacted negatively to the government's decision on the tax increase and hence using a football field would thus make a perfect anchor candidate for a compelling narrative. She finds out that her crowd fits within half of the field: an easy to grasp concept. Now, Lisa has her full sequence of relations, consisting of re-expressing the debt in terms of years of work with a human as a visual unit, organized in a football field, an easily relatable structured space.

\section{Discussion and Research Agenda}

This analysis of design practices and strategies for concrete scales raises many empirical questions including the effectiveness and efficiency at accurately depicting complex measures and how they affect an observer's construction of accurate mental models of scales in the long run. Our work examines design-related questions regarding the choice and adequacy of techniques, anchors and containers, and we provide tools to guide designers in making informed decisions in their design processes. We believe that there is no general answer to what makes an optimal concrete scale, since many factors come into play. As illustrated in our scenario, the topic itself, the message to convey, the context, the audience, pragmatic aspects and constraints may play a role in the final design. However, we have opened up questions that would help shed light on scale cognition and communication, which we propose as the next steps in the research agenda in this area.

How do people conceive and communicate complex measures? Despite the large size of our corpus, it was difficult to ascertain highlyspecific trends and practices from the material due to the variety in the themes and measures. More studies, like that of Ma [19], should examine what people mentally conceive or draw when asked to represent a given complex measure. Such studies would provide great insights on how to push empirical research further on using specific strategies or practices under controlled conditions.

What defines familiarity? The notion of familiarity is tightly linked with our ability to construct mental models of scale through cognitive mechanisms like anchor and adjustment or unitization. Yet, what defines familiarity to observers and how designers can select the right objects remains unclear. Even when we are familiar with an object, the question whether we can accurately recall its absolute dimensions is far from being certain. This would provide an interesting avenue to investigate, especially when our memory and accuracy of various anchors seem to be distorted from reality in the first place.

Empirical comparison. From this work also emerged the need for empirical evaluations that examine the multiple effects of various strategies, conversions, sets of anchors, containers and units on scale cognition. Questions such as "what makes a good set of anchor points?", "at which magnitude granularity should we add anchors?", could be answered with further exploration of this question.

\section{ConClusion}

This paper is the first attempt at examining the design space of concrete scales through a deconstruction and analysis of a large corpus depicting measures of extreme magnitudes and unfamiliar units. Informed by our extensive literature review and in-depth visual analysis, which encompasses scale cognition mechanisms in psychology, we outline the different types of measure relations and representation strategies that are commonly used. We also demonstrate our framework through several case studies. Lastly, we contribute design recommendations and pinpoint potential pitfalls that we hope will serve as reference tools for designers and researchers alike, and propose directions for further research in this area.

\section{ACKNOWLEDGMENTS}

We wish to thank Pierre Dragicevic and Jean-Daniel Fekete for valuable discussions on this work, Benjamin Bach, Jérémy Boy, Petra and Tobias Isenberg, Yvonne Jansen, Charles Perin and Wesley Willet for their feedback on early drafts of our paper, and to our anonymous reviewers for their comments and suggestions to improve this article 


\section{REFERENCES}

[1] 10000 Pennies. The national debt road trip, 2009. http://tinyurl. com/1000pennies [accessed 2013-07-31].

[2] J. Allik and T. Tuulmets. Occupancy model of perceived numerosity. Perception \& Psychophysics, 49(4):303-314, 1991.

[3] S. Bateman, R. Mandryk, C. Gutwin, A. Genest, D. McDine, and C. Brooks. Useful junk?: the effects of visual embellishment on comprehension and memorability of charts. In Proceedings of the 28th international conference on Human factors in computing systems, pages 2573-2582. ACM, 2010.

[4] K. Boeke. Cosmic View: The Universe in Forty Jumps. John Day Company, 1957.

[5] C. Eames and R. Eames. Powers of ten, 1977. http://www . powersof10.com/ [accessed 2013-07-31].

[6] Eatorama.com. Rethink your drinks, 2009. http://tinyurl.com/ drinkrethink [accessed 2013-07-31].

[7] R. K. Fulbright, S. C. Manson, P. Skudlarski, C. M. Lacadie, and J. C. Gore. Quantity determination and the distance effect with letters, numbers, and shapes: A functional $\mathrm{mr}$ imaging study of number processing. AJNR Am J Neuroradiol, 23:193-200, 2003.

[8] M. Gleicher, D. Albers, R. Walker, I. Jusufi, C. D. Hansen, and J. C. Roberts. Visual comparison for information visualization. Information Visualization, 10(4):289-309, oct 2011.

[9] M. Graham and J. Kennedy. A survey of multiple tree visualisation. Information Visualization, 9(4):235-252, Dec. 2010

[10] C. Huang and M. Huang. The scale of the universe 2, 2012. http: //htwins. net/scale2/ [accessed 2013-07-31].

[11] W. Javed and N. Elmqvist. Exploring the design space of composite visualization. In Proceedings of the IEEE Pacific Symposium on Visualization, pages 1-8, 2012.

[12] G. Jones, A. Taylor, and B. Broadwell. Estimating linear size and scale: Body rulers. Journal of Science Education, 3(11):1495-1509, 2009.

[13] M. Jones and A. Taylor. Developing a Sense of Scale: Looking Backward. Journal of Research in Science Teaching, 4(46):460-475, 2011.

[14] K. Karlstrom, et al. Informal geoscience education on a grand scale: The trail of time exhibition at grand canyon. Journal of Geoscience Education, 56(4):354-361, 2008.

[15] Kleptocracy. A visualization of united states debt, 2011. http:// usdebt.kleptocracy.us / [accessed 2013-07-31].

[16] S. M. Kosslyn. Understanding charts and graphs. Applied Cognitive Psychology, 3:185-225, 1989.

[17] G. Light, S. Swarat, E.-J. Park, D. Drane, E. Tevaarwerk, and T. Mason. Understanding undergraduate students' conceptions of a core nanoscience concept: Size and scale. In Proceedings of the Conference on Research in Engineering Education, pages 1441-1450, 2007.

[18] J. Ma. Scale ladders - communicating size and scale. Technical report, Exploratorium, 2007.

[19] J. Ma. Visitors' drawings of small. Technical report, Exploratorium, 2007.

[20] L. Micallef, P. Dragicevic, and J.-D. Fekete. Assessing the Effect of Visualizations on Bayesian Reasoning Through Crowdsourcing. IEEE Transactions on Visualization and Computer Graphics, Oct. 2012.

[21] M. Miner and B. Silleck. Cosmic voyage, 1996. http: //tinyurl.com/ imax-cosmicvoyage [accessed 2013-07-31].

[22] NASA. Astronomy picture of the day - all the water on europa, 2012 http: //tinyurl.com/water-europa [accessed 2013-07-31]
[23] O. Neurath. International Picture Language. Department of Typography \& Graphic Comm., 1980.

[24] A. Nieman. Concrete vs abstract visualisation: the real world as a canvas for data visualisation. In Proceedings of ADS-VIS2011: Making visible the invisible: Art, design and science in data visualisation, pages 49-56, 2012.

[25] D. Obreschkow. Cosmic eye, 2012. http://tinyurl.com/cosmiceye [accessed 2013-07-31].

[26] J. Parker. Using Google Earth to teach the magnitude of deep time. Journal of College Science Teaching. Journal of College Science Teaching, 5(40):23-27, 2011

[27] R. D. Portugal and B. F. Svaiter. Weber-fechner law and the optimality of the logarithmic scale. Minds Mach., 21(1):73-81, Feb. 2011.

[28] H. Rijgersberg and J. Top. Unitdim: an ontology of physical units and quantities. year [ca. 2004], 2004.

[29] H. Rijgersberg, M. Wigham, and J. Top. How semantics can improve engineering processes: A case of units of measure and quantities. Advanced Engineering Informatics, 25(2):276-287, 2011.

[30] Sciencenter. Sagan planet walk, 2003. http://www.sciencenter. org/saganpw/ [accessed 2013-07-31].

[31] M. Song and C. Quintana. Representing "too small to see" as "too small to see" with temporal representation. In Proceedings of the 2012 ACM annual conference on Human Factors in Computing Systems, CHI '12, pages 1441-1450, 2012.

[32] SugarStacks.com. Sugar stacks, 2009. http://www.sugarstacks. $\mathrm{com} /$ [accessed 2013-07-31]

[33] S. Swarat, G. Light, E. J. Park, and D. Drane. A typology of undergraduate students' conceptions of size and scale: Identifying and characterizing conceptual variation. Journal of Research in Science Teaching, 5(48):512-533, 2011.

[34] E. Szasz. Cosmic zoom, 1968. http://www.nfb.ca/film/cosmic_ zoom/ [accessed 2013-07-31].

[35] N. Tractinsky and J. Meyer. Chartjunk or goldgraph? effects of presentation objectives and content desirability on information processing. MIS Quarterly, 23(3):397-421, 1999.

[36] T. Tretter, M. Jones, T. Andre, A. Negishi, and J. Minogue. Conceptual Boundaries and Distances: Students' and Experts' Concepts of the Scale of Scientific Phenomena. Journal of Research in Science Teaching, 3(43):282-319, 2006

[37] T. Tretter, M. Jones, and J. Minogue. Accuracy of Scale Conceptions in Science: Mental Maneuverings across Many Orders of Spatial Magnitudes. Journal of Research in Science Teaching, 10(43):1061-1085, 2006.

[38] US Geologic Survey. How much water is there on, in, and above the earth?, 2012. http://ga.water.usgs.gov/edu/earthhowmuch. html [accessed 2013-07-31].

[39] V. Verma and A. Pang. Comparative flow visualization. IEEE Transactions on Visualization and Computer Graphics, 10(6):609-624, Nov. 2004

[40] Y. von Engelhardt. The Language of Graphics: A Framework for the Analysis of Syntax and Meaning in Maps, Charts and Diagrams. PhD thesis, Institute for Language, Logic and Computation, Universiteit van Amsterdam, 2002.

[41] C. Ware. Visual Thinking. Morgan Kaufmann, 2008. 